\title{
Merkel Cell Carcinoma Metastatic to Pleural Fluid: A Case Report
}

\author{
Ye-Young Rhee · Soo Hee Kim \\ Eun Kyung $\mathrm{Kim}^{1} \cdot$ Se Hoon $\mathrm{Kim}^{1}$ \\ Pathology Center, Seegene Medical Foundation, \\ Seoul; 'Department of Pathology, Severance \\ Hospital, Yonsei University College of Medicine, \\ Seoul, Korea
}

Received: October 11, 2017

Revised: November 7, 2017

Accepted: November 9, 2017

Corresponding Author

Se Hoon Kim, MD, PhD, FIAC

Department of Pathology, Severance Hospital,

Yonsei University College of Medicine, 50-1

Yonsei-ro, Seodaemun-gu, Seoul 03722, Korea

Tel: +82-2-2228-1769

Fax: +82-2-362-0860

E-mail: paxco@yuhs.ac
Merkel cell carcinoma (MCC) is a rare aggressive neuroendocrine carcinoma of the skin that shows locoregional or distant metastasis. Metastasis of MCC to body cavity effusion is extremely rare; only three cases have been reported so far. Metastatic MCC in effusion cytology shows small blue round cells with fine stippled chromatin like other small blue round cell tumors such as small cell lung carcinoma or lymphoma. The diagnosis of metastatic MCC can grant patients good chances at recently advanced therapeutic options. Here, we present a case of metastatic MCC to pleural effusion with characteristic single file-like pattern.

Key Words: Carcinoma, Merkel cell; Neoplasm metastasis; Pleural fluid; Liquid-based cytology
Merkel cell carcinoma (MCC) is an uncommon aggressive neuroendocrine tumor of the skin that occurs primarily in the elderly or immunosuppressed individuals and often shows locoregional or distant metastasis. ${ }^{1-3}$ Toker first reported it as "trabecular carcinoma" in 1972, ${ }^{4}$ however, MCC has not been investigated as actively as other skin tumors due to its rarity and uncertain etiology until recently when diagnostic tools including immunohistochemistry have improved and polyomavirus has been identified to be associated with the tumor. ${ }^{2,5}$ Although the incidence of MCC is increasing and locoregional recurrence or metastasis has become common, distant metastases of MCC especially to body cavity effusions are extremely rare. There are only three case reports of metastatic MCC to effusions: two to pleural fluid and one to ascites. ${ }^{6-8}$ Here, we report the first case of metastatic MCC to pleural effusion in Korea.

\section{CASE REPORT}

This study was approved by the Institutional Review Board of Severance Hospital with a waiver of informed consent (IRB No. 4-2017-0634).

A 68-year-old female with a medical history of hypertension was diagnosed with MCC in the left buttock 2 years ago at an outside hospital. The patient underwent excision of the tumor with inguinal lymph node dissection. Local recurrence occurred after one year, leading to additional left inguinal lymph node dissection. According to the positron emission tomography, metastases to the regional, intra-abdominal, and right axillary lymph nodes were identified. The patient was treated with three cycles of etoposide and cisplatin (EP) chemotherapy and radiotherapy in the left leg, para-aortic lymph node, and right inguinal lymph node. The patient was transferred to our hospital due to bone metastasis. Even after five cycles of cyclophosphamide, doxorubicin and vincristine (CAV), the disease progressed and the patient received first intraperitoneal chemotherapy. The patient was admitted to the hospital for fever, diarrhea, poor oral intake, and pancytopenia. Metastasis in a small-to-borderline sized lymph node in the left axilla and bilateral pleural effusion (Fig. 1) were identified.

Surgically excised specimen stained with hematoxylin and eosin presented blue round cell tumor with infiltrative growth, showing sheets, clusters, rows or balls of tumor cells with scanty cytoplasm, hyperchromatic nuclei, fine stippled or smudged chromatin and occasional mitoses (Fig. 2A-D). Immunohistochemical staining showed the tumor cells positive for cytokeratin 20 (CK20), CD56, chromogranin, and synaptophysin (Fig. 2C, D). 


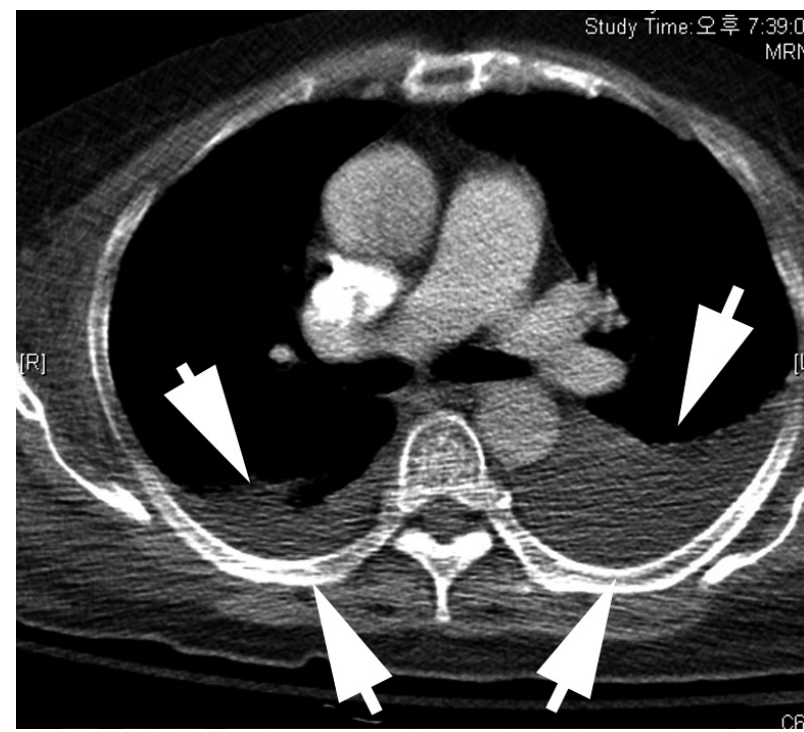

Fig. 1. Chest computed tomography (CT) with cell block findings. The chest CT shows marked pleural effusion (arrows).
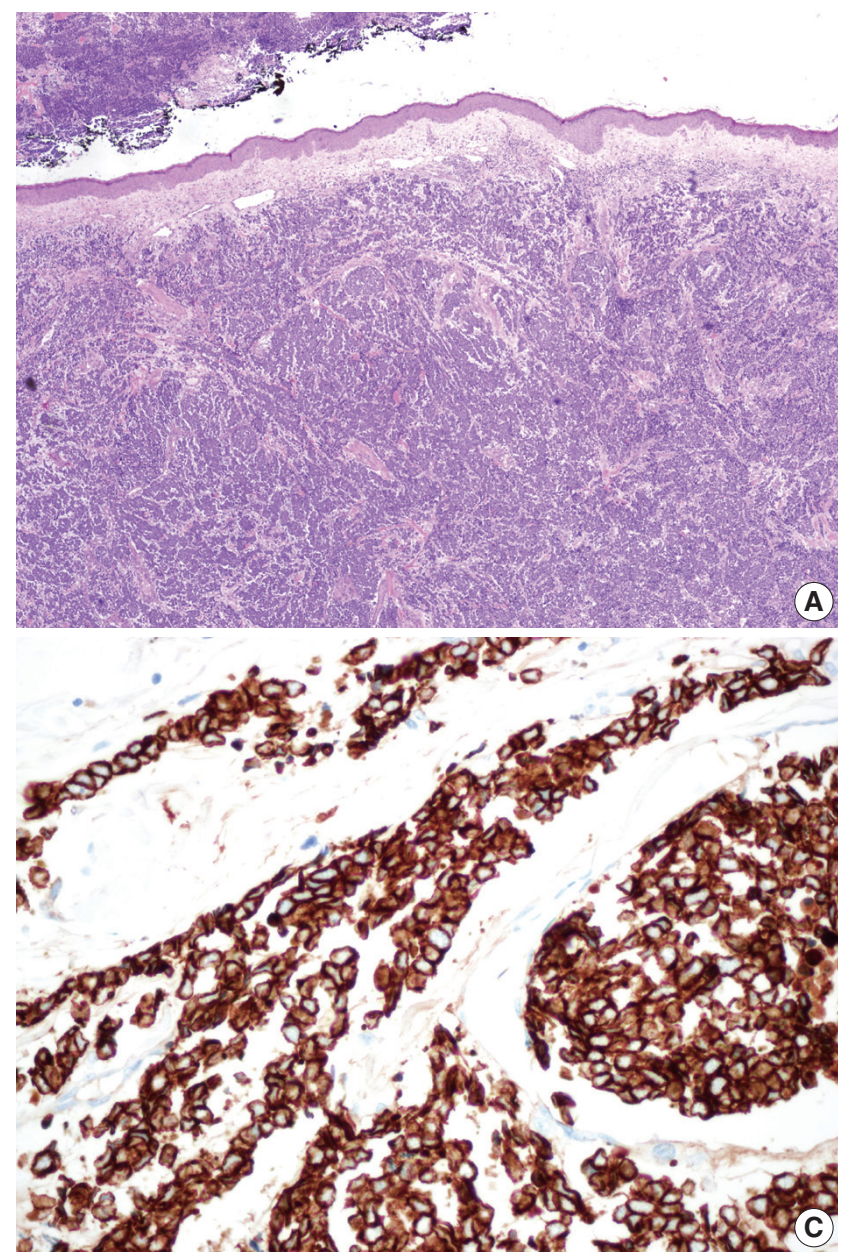

Liquid based cytology (LBC; SurePath, BD Diagnostics, Burlington, NC, USA) (Fig. 3A) and cell block of pleural effusion (Fig. 3B) revealed malignant small blue round cells scattered, clustered, or arranged in a single file. Round to oval tumor cells showed hyperchromatic nuclei with fine granular salt and pepper chromatin, scant cytoplasm, occasional mitotic figures, and occasional nuclear molding (Fig. 3A). Immunohistochemical staining on the cell block also showed the tumor cells immunoreactive for CK20, CD56, chromogranin, and synaptophysin (Fig. 3C, D).

\section{DISCUSSION}

MCC is a rare and highly aggressive skin carcinoma often seen in the head and neck region. ${ }^{5}$ The increasing incidence ${ }^{1,9,10}$ and recent new findings about risk factors, especially the identification of polyomavirus infection, ${ }^{2}$ stimulated the investigation of the etiology, pathogenesis and treatment options. ${ }^{3,5}$ Although
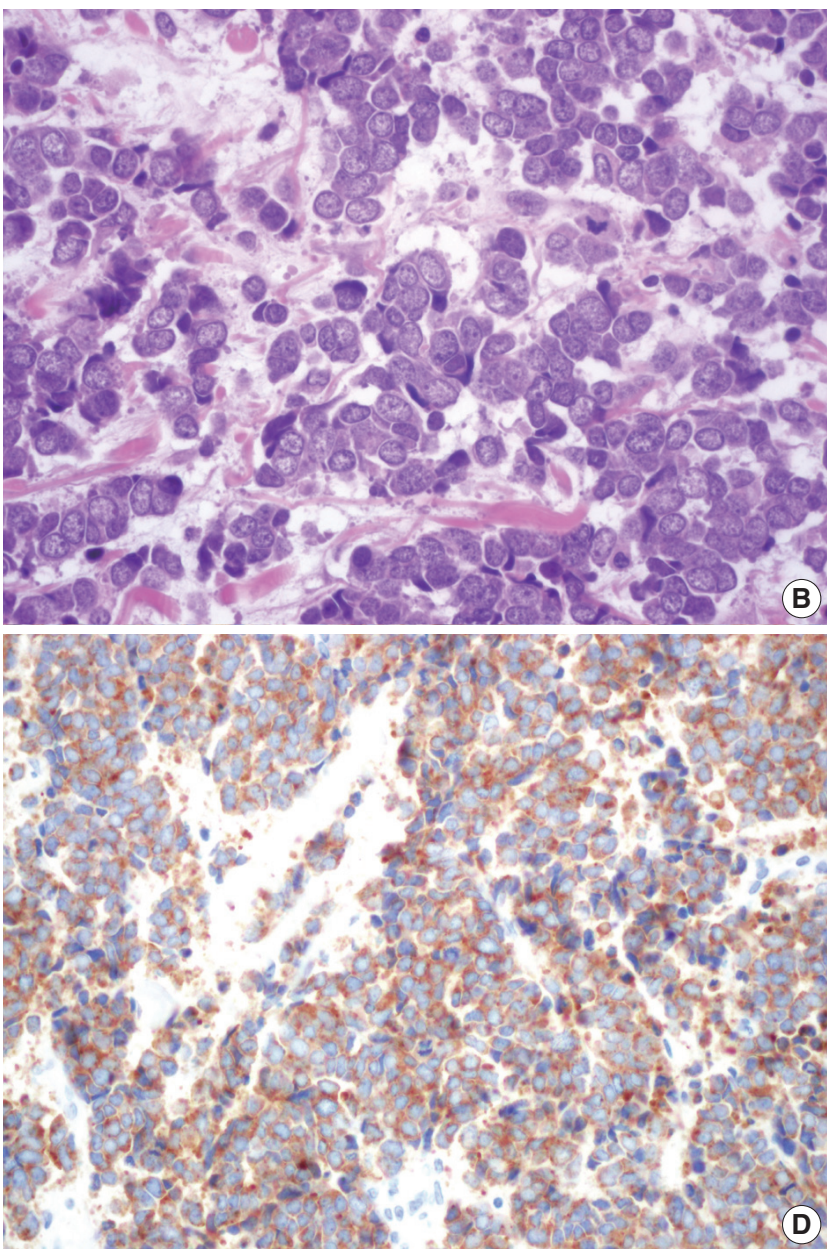

Fig. 2. Microscopic findings of the surgical specimen. Blue small round cell tumor with infiltrative growth (A) arranged in sheets, clusters, rows or balls, showing hyperchromatic nuclei, fine stippled or smudged chromatin and occasional mitoses (B). Immunohistochemical staining of cytokeratin 20 (C) and synaptophysin (D) show positive reaction in Merkel cell carcinoma cells. 


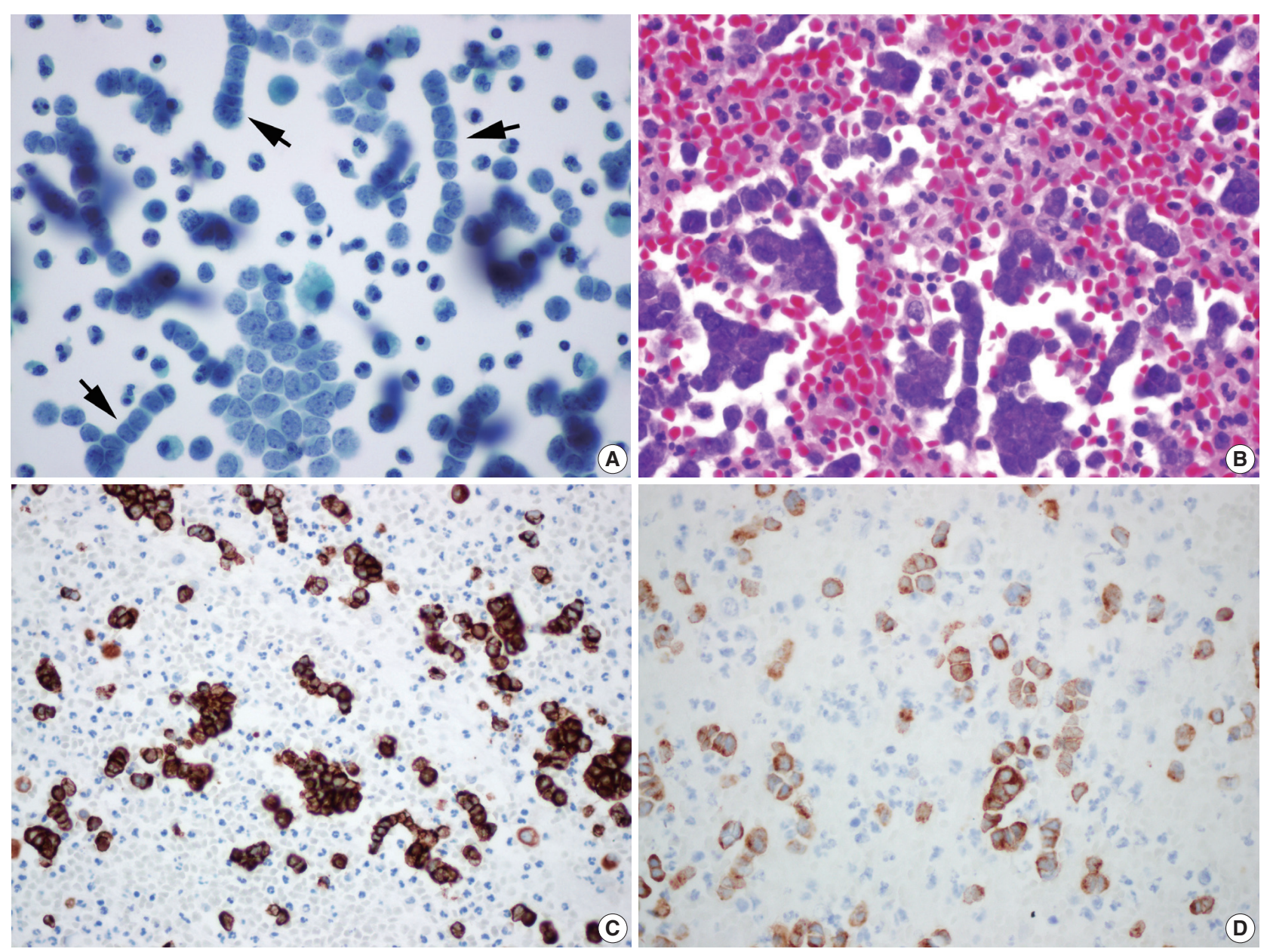

Fig. 3. (A) Liquid based cytology (BD Surepath) of the pleural effusion (Papanicolaou stain). Malignant small blue round cells are scattered, clustered or arranged with hyperchromatic nuclei, fine granular salt and pepper chromatin, scant cytoplasm, and occasional mitotic figures. Several single file patterns with nuclear molding are characteristic (arrows). (B) The cell block findings are similar to those in liquid based cytology's. Immunohistochemical staining of cytokeratin 20 (C) and synaptophysin (D) in the cell block of pleural effusion.

most patients present with localized tumors or with locoregional metastasis including lymph node metastasis without a primary MCC, ${ }^{11}$ distant metastases have also been reported. According to a single institution data, the clinical stage III (positive regional lymph node) and IV (distant metastatic disease) were $24 \%$ and $6 \%$, respectively. ${ }^{12}$

We reported a rare case of metastatic MCC to pleural effusion that had not been reported in Korea before. So far, only two cases of pleural metastasis of MCC have been reported worldwide. The first case was a metastasis of left great toe MCC to the pleural fluid, confirmed by electron microscopy. ${ }^{6}$ The second case was a metastasis of right buttock MCC to the pleural fluid, confirmed by immunohistochemical staining of CK20. ${ }^{7}$ Both cases showed small blue round cell clusters with fine granular chromatin in the pleural fluid as in our case. MCC stains positive for CK20 (a dot-like perinuclear positivity pattern), other epithelial markers (AE1/AE3, epithelial membrane antigen, and CAM 5.2), and neuroendocrine markers (chromogranin, CD56, synaptophysin, and somatostatin). ${ }^{5}$ We confirmed our diagnosis by immunoreactivity of the tumor to CK20, CD56, chromogranin, and synaptophysin both in surgical specimen and cytology cell block.

The diagnosis of metastatic MCC to body cavity effusion is easy to be missed because of its rarity and similarity of morphology to other small round cell tumors. Microscopic findings of the pleural effusion cytology of metastatic MCC can be confused with metastasis of other malignant neoplasm showing small blue round cells: small cell lung carcinoma, lymphoma, malignant melanoma, etc. However, immunohistochemical markers such as CK20, CD56, chromogranin, and synaptophysin would be helpful.

In this case, multiple single file patterns with cytological 
molding were dominant in both LBC and cell block. This identification of multiple single file patterns is a novel finding that has not been described in previous reports. ${ }^{6,7}$ It is more characteristic in LBC and may therefore be a helpful cytological clue in the era of LBC. The detection of hidden MCC presented only as distant metastasis in effusion could give patients good chances at cure because therapeutic options for MCC have increased recently: chemotherapy, immunotherapy, radiotherapy and targeted therapy. ${ }^{13}$

\section{Conflicts of Interest}

No potential conflict of interest relevant to this article was reported.

\section{REFERENCES}

1. Hodgson NC. Merkel cell carcinoma: changing incidence trends. J Surg Oncol 2005; 89: 1-4.

2. Feng H, Shuda M, Chang Y, Moore PS. Clonal integration of a polyomavirus in human Merkel cell carcinoma. Science 2008; 319: 1096-100.

3. Pulitzer MP, Amin BD, Busam KJ. Merkel cell carcinoma: review. Adv Anat Pathol 2009; 16: 135-44.

4. Toker C. Trabecular carcinoma of the skin. Arch Dermatol 1972; 105: 107-10.
5. Pulitzer M. Merkel cell carcinoma. Surg Pathol Clin 2017; 10: 399408.

6. Watson CW, Friedman KJ. Cytology of metastatic neuroendocrine (Merkel-cell) carcinoma in pleural fluid: a case report. Acta Cytol 1985; 29: 397-402.

7. Payne MM, Rader AE, McCarthy DM, Rodgers WH. Merkel cell carcinoma in a malignant pleural effusion: case report. Cytojournal 2004; $1: 5$.

8. Policarpio-Nicolas ML, Avery DL, Hartley T. Merkel cell carcinoma presenting as malignant ascites: a case report and review of literature. Cytojournal 2015; 12: 19.

9. Fitzgerald TL, Dennis S, Kachare SD, Vohra NA, Wong JH, Zervos EE. Dramatic increase in the incidence and mortality from Merkel cell carcinoma in the United States. Am Surg 2015; 81: 802-6.

10. Robertson JP, Liang ES, Martin RC. Epidemiology of Merkel cell carcinoma in New Zealand: a population-based study. Br J Dermatol 2015; 173: 835-7.

11. Eng TY, Boersma MG, Fuller CD, et al. A comprehensive review of the treatment of Merkel cell carcinoma. Am J Clin Oncol 2007; 30: 624-36.

12. Allen PJ, Bowne WB, Jaques DP, Brennan MF, Busam K, Coit DG. Merkel cell carcinoma: prognosis and treatment of patients from a single institution. J Clin Oncol 2005; 23: 2300-9.

13. Barksdale SK. Advances in Merkel cell carcinoma from a pathologist's perspective. Pathology 2017; 49: 568-74. 